\title{
Associations of Urine Biomarkers with Kidney Function Decline in HIV-Infected and Uninfected Men
}

\author{
Simon B. Ascher ${ }^{a, b}$ Rebecca Scherzer ${ }^{a}$ Michelle M. Estrella ${ }^{a}$
}

\author{
Michael G. Shlipak ${ }^{a, c}$ Derek K. Ng ${ }^{d}$ Frank J. Palella ${ }^{e}$ Mallory D. Witt ${ }^{f}$ Ken Ho $^{g}$ \\ Michael R. Bennett ${ }^{\mathrm{h}}$ Chirag R. Parikh ${ }^{\mathrm{i}}$ Joachim H. Ix ${ }^{\mathrm{j}, \mathrm{k}}$ Vasantha Jotwani ${ }^{\mathrm{a}}$
}

\begin{abstract}
${ }^{a}$ Kidney Health Research Collaborative, Department of Medicine, San Francisco Veterans Affairs Medical Center and University of California, San Francisco, CA, USA; ${ }^{b}$ Department of Medicine, University of California, Los Angeles, CA, USA; ' Department of Epidemiology and Biostatistics, University of California, San Francisco, CA, USA; ${ }^{\mathrm{d} D e p a r t m e n t}$ of Epidemiology, Johns Hopkins Bloomberg School of Public Health, Baltimore, MD, USA; ${ }^{e}$ Division of Infectious Disease, Department of Medicine, Northwestern University Feinberg School of Medicine, Chicago, IL, USA; ${ }^{f}$ Department of Medicine, Los Angeles Biomedical Research Institute at Harbor-UCLA Medical Center, Torrance,

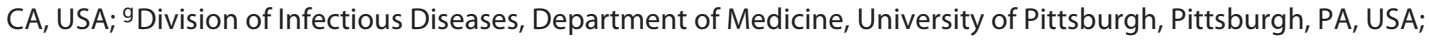
h Division of Nephrology and Hypertension, Cincinnati Children's Hospital Medical Center, Cincinnati, OH, USA; 'Division of Nephrology, Department of Medicine, Johns Hopkins School of Medicine, Baltimore, MD, USA; 'Division of Nephrology-Hypertension, University of California, San Diego, CA, USA; ${ }^{k}$ Nephrology Section, Department of Medicine, Veterans Affairs San Diego Healthcare System, San Diego, CA, USA
\end{abstract}

\section{Keywords}

HIV · Urine biomarker · Kidney damage ·

Alpha-1-microglobulin · Albuminuria

\begin{abstract}
Background: HIV-infected (HIV+) persons are at increased risk of chronic kidney disease, but serum creatinine does not detect early losses in kidney function. We hypothesized that urine biomarkers of kidney damage would be associated with subsequent changes in kidney function in a contemporary cohort of HIV+ and HIV-uninfected (HIV-) men. Methods: In the Multicenter AIDS Cohort Study, we measured baseline urine concentrations of 5 biomarkers from 2009 to 2011 in 860 HIV+ and 337 HIV-men: albumin, alpha-1-microglobulin (a1m), interleukin-18 (IL-18), kidney injury molecule-1 (KIM-1), and procollagen type III N-terminal propep-
\end{abstract}

๑ 2019 S. Karger AG, Basel

E-Mail karger@karger.com

www.karger.com/ajn tide (PIIINP). We evaluated associations of urine biomarker concentrations with annual changes in estimated glomerular filtration rate (eGFR) using multivariable linear mixed models adjusted for demographics, traditional kidney disease risk factors, HIV-related risk factors, and baseline eGFR. Results: Over a median follow-up of 4.8 years, the average annual eGFR decline was $1.42 \mathrm{~mL} / \mathrm{min} / 1.73 \mathrm{~m}^{2} /$ year in HIV+ men and $1.22 \mathrm{~mL} / \mathrm{min} / 1.73 \mathrm{~m}^{2} /$ year in HIV- men. Among $\mathrm{HIV}+$ men, the highest vs. lowest tertiles of albumin $\left(-1.78 \mathrm{~mL} / \mathrm{min} / 1.73 \mathrm{~m}^{2} /\right.$ year, $95 \% \mathrm{Cl}-3.47$ to -0.09$)$ and $\mathrm{a} 1 \mathrm{~m}$ $\left(-2.43 \mathrm{~mL} / \mathrm{min} / 1.73 \mathrm{~m}^{2} /\right.$ year, $95 \% \mathrm{Cl}-4.14$ to -0.73$)$ were each associated with faster annual eGFR declines after multivariable adjustment. Among HIV-men, the highest vs. lowest tertile of a $1 \mathrm{~m}\left(-2.49 \mathrm{~mL} / \mathrm{min} / 1.73 \mathrm{~m}^{2} /\right.$ year, $95 \% \mathrm{Cl}-4.48$ to -0.50$)$ was independently associated with faster annual eGFR decline. Urine IL-18, KIM-1, and PIIINP showed no independent associations with eGFR decline, regardless of HIV
Vasantha Jotwani, MD

Kidney Health Research Collaborative, Department of Medicine San Francisco Veterans Affairs Medical Center and University of California 4150 Clement Street, Bldg 2, Rm 145, San Francisco, CA 94121 (USA)

E-Mail Vasantha.Jotwani@ucsf.edu 
serostatus. Conclusions: Among HIV+ men, higher urine albumin and $\alpha 1 \mathrm{~m}$ are associated with subsequent declines in kidney function, independent of eGFR. @ $2019 \mathrm{~S}$. Karger AG, Basel

\section{Introduction}

In the era of combination antiretroviral therapy, HIV infection has become a chronic condition in which agerelated comorbidities have become more common and may occur at an earlier age $[1,2]$. Compared to uninfected individuals, HIV-infected individuals are at higher risk of developing proteinuria, chronic kidney disease (CKD), and end-stage kidney disease [3-5]. In addition, $\mathrm{HIV}$-infected individuals are at increased risk of cardiovascular disease, heart failure, and mortality, which are all endpoints strongly associated with CKD [6].

Clinical practice guidelines by the HIV Medicine Association of the Infectious Diseases Society of America recommend monitoring for kidney disease with serum creatinine and urine albumin, but both tests have limitations [7]. Serum creatinine, which is used to calculate an estimated glomerular filtration rate (eGFR), lacks sensitivity for detecting early reductions in kidney function in HIVinfected individuals $[4,8]$. In addition, substantial kidney injury can occur prior to a measurable reduction in kidney function. Urine albumin is the most common clinically used kidney injury marker and reflects endothelial damage to the glomerulus. However, kidney tubular epithelial cells are a long-term reservoir site for HIV infection, and kidney biopsy studies have shown that tubular injury and interstitial fibrosis are strong predictors of CKD progression in HIV-infected and uninfected populations [9-14].

Urine alpha-1-microglobulin $(\alpha 1 \mathrm{~m})$, a marker of proximal tubular dysfunction, interleukin-18 (IL-18), kidney injury molecule-1 (KIM-1), markers of proximal tubular injury, and procollagen type III N-terminal propeptide (PIIINP), a marker of renal interstitial fibrosis, are promising urine biomarkers of kidney tubule health. We previously found that HIV-infected women have significantly higher urine a1m, IL-18, KIM-1, and PIIINP levels compared to uninfected women [15-17]. Furthermore, we observed among HIV-infected women that higher urine albumin, $\alpha 1 \mathrm{~m}$, IL-18, and KIM-1 levels were each associated with faster eGFR decline over 8 years of follow-up $[18,19]$. Whether or not levels of these urine biomarkers are associated with kidney function decline in a more contemporary cohort, including HIV-infected men, are unclear.
In this longitudinal study of HIV-infected and uninfected men, we evaluated associations of levels of urine biomarkers of glomerular injury (albuminuria), tubular dysfunction ( $(\mathrm{lm})$, tubular injury (IL-18 and KIM-1), and interstitial fibrosis (PIIINP) with subsequent changes in kidney function. We hypothesized that more extensive kidney damage, as measured by higher urine biomarker concentrations, would be independently associated with faster eGFR decline.

\section{Materials and Methods}

\section{Study Design}

The Multicenter AIDS Cohort Study (MACS) is one of the largest cohort studies of HIV-infected individuals in the United States. The design and methods have been described previously [20]. In brief, 6,972 men who have sex with men with HIV infection or at high risk for HIV infection volunteered for enrollment in 19841985, 1987-1990, and 2001-2003 from 4 sites: Baltimore, Chicago, Los Angeles, and Pittsburgh. MACS participants attend semiannual visits for follow-up evaluations that include a standardized questionnaire, a physical examination, and collection of laboratory specimens.

The MACS Kidney Study was designed as a nested cohort to investigate the development and progression of kidney disease among HIV-infected and uninfected men. This longitudinal study included all $883 \mathrm{HIV}$-infected men with urine samples collected between October 1, 2009, and September 30, 2011, and a random sample of 350 uninfected men with available urine specimens from this time period. For the present study, we excluded 36 individuals who did not have a follow-up serum creatinine measurement, leaving a final analytic sample of 1197 (860 HIV-infected and $337 \mathrm{HIV}$ uninfected).

\section{Predictors}

We measured urine concentrations of the following biomarkers: albumin, $\alpha 1 \mathrm{~m}, \mathrm{IL}-18$, KIM-1, and PIIINP. All biomarkers were measured at the Cincinnati Children's Hospital Medical Center Biomarker Laboratory. Details regarding the selected commercial assays and inter-assay coefficients of variation are shown in online supplemental Table 1 (for all online suppl. material, see www.karger.com/doi/10.1159/000502898). Urine specimens were refrigerated immediately after collection and centrifuged at 5,000 $\mathrm{g}$ to remove cellular debris. The supernatant was aliquoted into $1-\mathrm{mL}$ vials and then stored at $-80^{\circ} \mathrm{C}$ until biomarker measurement without prior freeze-thaw. Laboratory personnel performing the biomarker assays were blinded to participants' clinical information.

\section{Outcomes}

The primary outcome was annual mean change in eGFR, expressed in $\mathrm{mL} / \mathrm{min} / 1.73 \mathrm{~m}^{2}$ per year. We calculated eGFR using the 2009 CKD Epidemiology Collaboration creatinine equation [21]. Serum creatinine was measured semi-annually at the clinical labs of each MACS site using the modified Jaffe method, traceable to isotope dilution mass spectrometry. Our secondary outcomes included: (1) incident CKD, defined as an eGFR $<60 \mathrm{~mL} / \mathrm{min} / 1.73 \mathrm{~m}^{2}$ on 2 consecutive visits separated by at least 3 months with at least 
$1 \mathrm{~mL} / \mathrm{min} / 1.73 \mathrm{~m}^{2}$ per year decline among persons with baseline eGFR $>60 \mathrm{~mL} / \mathrm{min} / 1.73 \mathrm{~m}^{2}$ and (2) annual eGFR decline $\geq 5 \%$ of the baseline eGFR.

\section{Covariates}

Demographics, kidney disease risk factors, and HIV-related parameters were assessed at the baseline visit, except where noted below, and included in all multivariable models. Demographic variables included age and race/ethnicity. Metabolic health variables included diabetes mellitus (defined as fasting glucose $\geq 126 \mathrm{mg} / \mathrm{dL}$, hemoglobin $\mathrm{A}_{1 \mathrm{c}} \geq 6.5 \%$, or self-reported history of diabetes and diabetes medication use) and body mass index. Cardiovascular health variables included systolic and diastolic blood pressure, hypertension (defined as systolic blood pressure $>140 \mathrm{~mm} \mathrm{Hg}$, diastolic blood pressure $>90 \mathrm{~mm} \mathrm{Hg}$, or self-reported history of hypertension and antihypertensive medication use), self-reported cardiovascular disease, and levels of low- and highdensity lipoprotein cholesterol. Behavioral health risk factors included cigarette smoking status (current, past, or never), current heroin use, hepatitis $\mathrm{C}$ virus (HCV) infection (confirmed by detectable plasma HCV RNA following a positive HCV antibody result). HIV infection characteristics included CD4 T-lymphocyte count $/ \mathrm{mm}^{3}$ (nadir, baseline, and time-updated), plasma HIV-1 RNA level (peak, baseline, and time-updated), history of clinical AIDS diagnosis, current TDF use, and other highly active antiretroviral therapy (HAART) use.

\section{Statistical Analysis}

The percentage of missing observations for each baseline covariate ranged from $<1$ to $28 \%$. While all participants had eGFR measured at baseline, the percentage of missing eGFR increased over time, from $11 \%$ at year 1 to $53 \%$ at year 5 . Because $24 \%$ of urine a $1 \mathrm{~m}$ values were below the detectable limit of the assay $(0.53 \mathrm{mg} /$ $\mathrm{dL}$ ), we used a left-censored Tobit regression model to impute undetectable $a 1 \mathrm{~m}$ values. For all other variables, we used pattern mixture models with the neighboring-case missing values method to impute missing data, using 100 imputations to ensure high relative efficiency and reliable estimation [22-24]. This method is wellsuited to handle longitudinal data with dropout patterns that may not represent data that are missing at random. Imputation models included eGFR as well as all exposures and covariates listed above.

We compared demographic and baseline characteristics by HIV serostatus using $\chi^{2}$ and Mann-Whitney $U$ tests for categorical and continuous variables, respectively. We analyzed levels of urine biomarkers as continuous variables (log-transformed to achieve normality of right-skewness) and as categorical variables divided into tertiles. We also analyzed urine albumin as a dichotomous variable using the standard clinical cut-point (albumin-to-creatinine ratio $[\mathrm{ACR}] \leq 30 \mathrm{vs}$. $>30 \mathrm{mg} / \mathrm{g}$ ). We modeled the associations of each urine biomarker with annual mean change in eGFR using linear mixed effect models. All models were constructed separately by HIV serostatus. We accounted for serial within-subject correlations using a first-order autoregressive moving average covariance structure and controlled for baseline eGFR and follow-up time so that coefficients would be interpretable as annual changes in eGFR attributable to each covariate [25]. We estimated adjusted risk ratios for each two-fold higher level of each baseline urine biomarker with incident CKD and annual eGFR decline $\geq 5 \%$ using Poisson regression with a robust variance estimator [26]. We adjusted for urine creatinine in all models to correct for tonicity.

Urine Biomarkers and Kidney Function Decline in HIV
To determine whether levels of individual urine biomarkers were independently associated with study outcomes, multivariable models were adjusted for demographics, traditional kidney disease risk factors, and HIV-related risk factors (as listed above in the Covariates section). We also modeled all biomarkers in combination and evaluated all pairwise and 3-way biomarker combinations in order to identify markers that were simultaneously statistically significant. We performed interaction testing to evaluate whether biomarker level associations with change in eGFR differed by TDF use. Because HIV infection may have direct effects on kidney tubular health and with eGFR, we also performed interaction testing to evaluate whether biomarker level associations with change in eGFR differed by HIV RNA levels. Based on observed findings, we additionally performed interaction testing to evaluate whether urine albumin associations varied by levels of $\alpha 1 \mathrm{~m}$.

All analyses were performed using SAS version 9.4 (SAS Institute, Inc., Cary, NC, USA).

\section{Results}

At baseline, the median age was 52 years among the $860 \mathrm{HIV}$-infected men and 54 years among the 337 uninfected men (Table 1). Compared to uninfected men, HIVinfected men had a higher baseline prevalence of eGFR $<60 \mathrm{~mL} / \mathrm{min} / 1.73 \mathrm{~m}^{2}$ (9 vs. $\left.4 \%, p<0.001\right)$ and ACR $>30 \mathrm{mg} / \mathrm{g}(17 \mathrm{vs.} \%$, $p<0.001)$. Among the HIV-infected men, $85 \%$ were using HAART and $66 \%$ were using TDF; the median CD4 lymphocyte count was 576 cells $/ \mathrm{mm}^{3}$, and $78 \%$ had undetectable HIV RNA levels. Over a median follow-up period of 4.8 years (interquartile range 4.4-5.3), the average rate of annual eGFR decline was $1.42 \mathrm{~mL} / \mathrm{min} / 1.73 \mathrm{~m}^{2}$ per year (95\% CI $1.31-1.53$ ) in $\mathrm{HIV}$-infected men and $1.22 \mathrm{~mL} / \mathrm{min} / 1.73 \mathrm{~m}^{2}$ per year (95\% CI 0.74-1.70) in uninfected men.

In unadjusted analyses among the HIV-infected men, higher baseline urine albumin and $\alpha 1 \mathrm{~m}$ concentrations were associated with faster annual eGFR declines (Table 2). There was minimal attenuation of these associations after multivariable adjustment for traditional and HIVrelated kidney disease risk factors. When biomarker levels were analyzed as continuous variables, each two-fold higher level of urine albumin at baseline was associated with a $0.58 \mathrm{~mL} / \mathrm{min} / 1.73 \mathrm{~m}^{2}$ per year faster rate of eGFR decline in multivariable-adjusted models. Compared to participants with baseline ACR $\leq 30 \mathrm{mg} / \mathrm{g}$, those with ACR $>30 \mathrm{mg} / \mathrm{g}$ had faster eGFR declines. In tertile analyses, the highest vs. lowest tertiles of baseline urine albumin and a $1 \mathrm{~m}$ were each associated with faster eGFR declines in multivariable-adjusted models. By contrast, IL-18, KIM-1, and PIIINP were not significantly associated with eGFR decline in unadjusted or adjusted analyses. When we modeled levels of all 5 urine biomarkers simultaneously, 
Table 1. Baseline characteristics by HIV status

\begin{tabular}{|c|c|c|c|}
\hline & $\mathrm{HIV}+(n=860)$ & $\mathrm{HIV}-(n=337)$ & $p$ value \\
\hline Age, years & $52(46-58)$ & $54(49-62)$ & $<0.001$ \\
\hline \multicolumn{4}{|l|}{ Race } \\
\hline African American & $264(31)$ & $94(28)$ & \multirow[t]{3}{*}{0.15} \\
\hline Caucasian & $526(61)$ & $224(66)$ & \\
\hline Other & $70(8)$ & $19(6)$ & \\
\hline Diabetes mellitus & $107(16)$ & $43(15)$ & 0.75 \\
\hline BMI, $\mathrm{kg} / \mathrm{m}^{2}$ & $26(24-30)$ & $27(24-32)$ & 0.015 \\
\hline Hypertension & $360(46)$ & $150(47)$ & 0.75 \\
\hline History of cardiovascular disease & $56(7)$ & $17(5)$ & 0.34 \\
\hline Systolic blood pressure, $\mathrm{mm} \mathrm{Hg}$ & $126(116-135)$ & $128(117-136)$ & 0.14 \\
\hline Diastolic blood pressure, $\mathrm{mm} \mathrm{Hg}$ & $78(71-84)$ & $78(71-84)$ & 0.64 \\
\hline $\mathrm{LDL}, \mathrm{mg} / \mathrm{dL}$ & $108(88-132)$ & $115(92-137)$ & 0.011 \\
\hline $\mathrm{HDL}, \mathrm{mg} / \mathrm{dL}$ & $45(38-55)$ & $50(41-60)$ & $<0.001$ \\
\hline \multicolumn{4}{|l|}{ Smoking } \\
\hline Current & $250(30)$ & $76(23)$ & \multirow[t]{3}{*}{0.05} \\
\hline Past & $378(45)$ & $170(52)$ & \\
\hline Never & $210(25)$ & $82(25)$ & \\
\hline HCV infection & $83(10)$ & $30(9)$ & 0.69 \\
\hline Current heroin use & $11(1)$ & $4(1)$ & 0.90 \\
\hline $\mathrm{eGFR}, \mathrm{mL} / \mathrm{min} / 1.73 \mathrm{~m}^{2}$ & $91(76-103)$ & $89(78-100)$ & 0.71 \\
\hline $\mathrm{eGFR}<60 \mathrm{~mL} / \mathrm{min} / 1.73 \mathrm{~m}^{2}$ & $74(9)$ & $12(4)$ & 0.002 \\
\hline $\mathrm{ACR}>30 \mathrm{mg} / \mathrm{g}$ & $142(17)$ & $28(8)$ & $<0.001$ \\
\hline HAART use & $723(85)$ & & \\
\hline TDF use & $555(66)$ & & \\
\hline NRTI use & $728(85)$ & & \\
\hline NNRTI use & $401(47)$ & & \\
\hline PI use & $383(45)$ & & \\
\hline Current CD 4 , cells $/ \mathrm{mm}^{3}$ & $576(411-740)$ & & \\
\hline Nadir CD 4, cells $/ \mathrm{mm}^{3}$ & $287(177-408)$ & & \\
\hline History of AIDS & $123(14)$ & & \\
\hline Current HIV RNA >40 copies/mL & $188(22)$ & & \\
\hline Peak HIV RNA >100,000 copies/mL & $331(40)$ & & \\
\hline
\end{tabular}

Data are presented as median (IQR) or numbers (\%). $p$ values are from $\chi^{2}$ tests for categorical variables and Mann-Whitney $\mathrm{U}$ tests for continuous variables.

AIDS, acquired immunodeficiency syndrome; eGFR, estimated glomerular filtration rate; HAART, highly active antiretroviral therapy; HDL, high-density lipoprotein cholesterol; LDL, low-density lipoprotein cholesterol; NRTI, nucleoside reverse transcriptase inhibitor; NNRTI, non-nucleoside reverse transcriptase inhibitor; PI, protease inhibitor; TDF, tenofovir disoproxil fumarate; BMI, body mass index; HCV, hepatitis C virus; ACR, albumin-to-creatinine ratio.

higher urine albumin remained associated with faster eGFR decline $\left(-0.61 \mathrm{~mL} / \mathrm{min} / 1.73 \mathrm{~m}^{2}\right.$ annually per twofold higher urine albumin, $95 \% \mathrm{CI}-1.13$ to -0.09 ), whereas the associations of levels of other markers, including a $1 \mathrm{~m}$, with eGFR were attenuated and had weaker associations that did not reach statistical significance.

We next modeled associations of levels of urine biomarkers with annual changes in eGFR among HIV-uninfected men. In unadjusted analyses, higher baseline urine albumin and $\alpha 1 \mathrm{~m}$ concentrations were each associated with faster annual eGFR declines (Table 3). After multi- variable adjustment, ACR $>30$ vs. $\leq 30 \mathrm{mg} / \mathrm{g}$ and the highest vs. lowest tertile of $\alpha 1 \mathrm{~m}$ remained significantly associated with faster eGFR declines. When modeled as a continuous variable, each two-fold higher $a 1 \mathrm{~m}$ at baseline also remained independently associated with faster eGFR declines. In models including levels of all 5 urine biomarkers simultaneously, only a $1 \mathrm{~m}$ remained associated with faster eGFR decline $(-0.86,95 \% \mathrm{CI}-1.58$ to $-0.15 \mathrm{~mL} /$ $\mathrm{min} / 1.73 \mathrm{~m}^{2}$ annually per two-fold higher $\alpha 1 \mathrm{~m}$ ).

To explore effect modification between kidney tubule dysfunction and glomerular injury, we evaluated whether 
Table 2. Association of urine biomarkers with annual eGFR change in HIV+ MACS participants

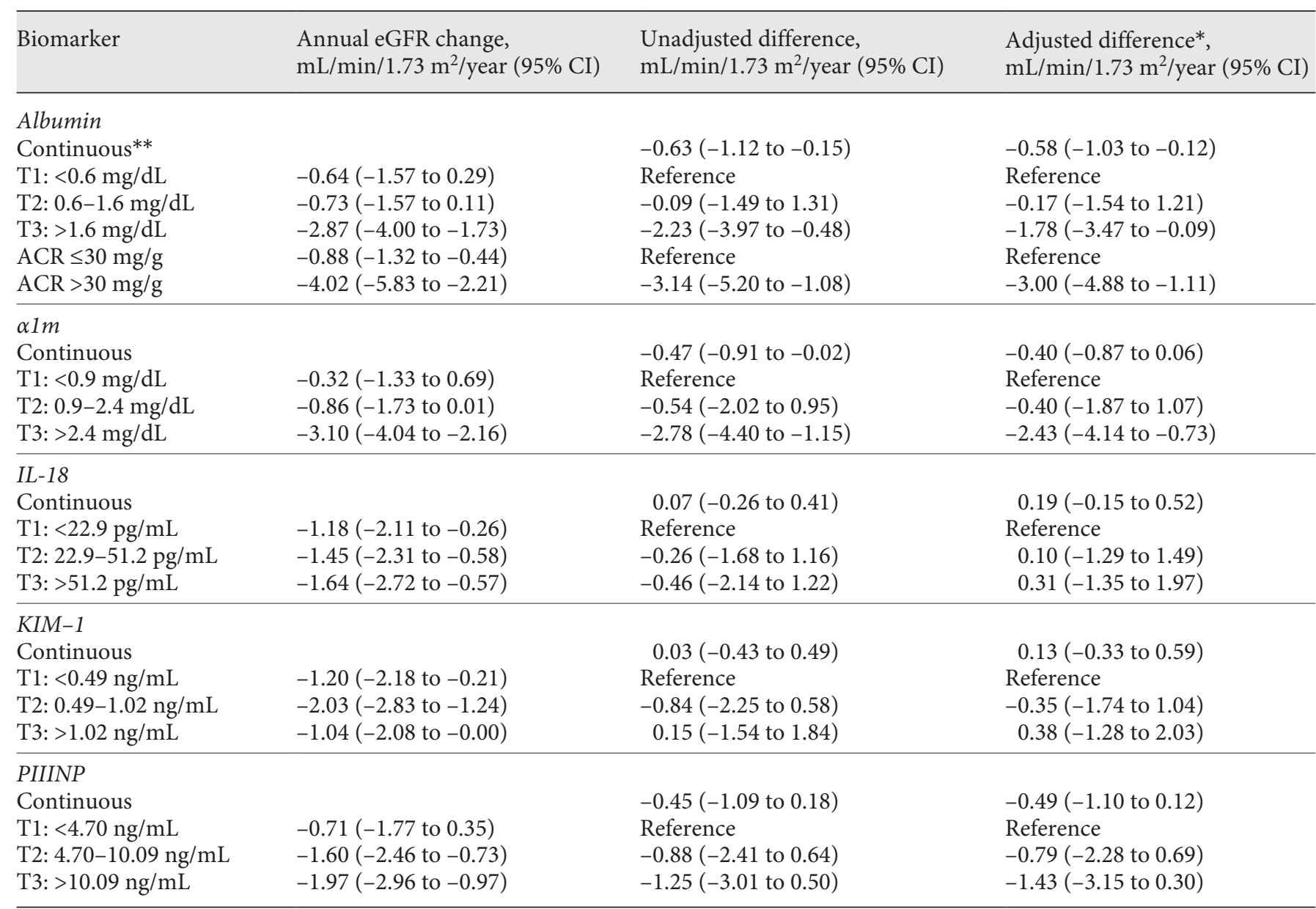

Biomarkers modeled individually, not jointly.

* Adjusted for age, race/ethnicity, urine creatinine, baseline eGFR, diabetes mellitus, systolic and diastolic blood pressure, hypertension, cardiovascular disease, low- and high-density lipoprotein cholesterol levels, triglyceride level, body mass index, waist circumference, cigarette smoking status, serum albumin, current heroin use, HCV infection, current CD4 lymphocyte count, plasma HIV-1 RNA level, history of clinical AIDS diagnosis, TDF use, and highly active antiretroviral therapy use.

** Continuous predictors are modeled per two-fold higher level of biomarker at baseline.

ACR, albumin-to-creatinine ratio; a1m, alpha-1-microglobulin; eGFR, estimated glomerular filtration rate; IL-18, interleukin-18; KIM-1, kidney injury molecule-1; PIIINP, procollagen type III N-terminal propeptide; MACS, Multicenter AIDS Cohort Study; HCV, hepatitis $\mathrm{C}$ virus.

urine levels of $\alpha 1 \mathrm{~m}$ modified the association of urine albumin with changes in eGFR. Among men who had simultaneously elevated levels of urine albumin and $\alpha 1 \mathrm{~m}$ (Fig. 1, online suppl. Table 2), rates of eGFR decline were substantially faster compared to those with lower levels ( $p$ value for interaction: 0.09 in HIV-infected and 0.03 in HIV-uninfected). Among HIV-infected men, the adjusted difference in annual eGFR decline was -3.02 (95\% CI -4.81 to -1.23$) \mathrm{mL} / \mathrm{min} / 1.73 \mathrm{~m}^{2}$ per year faster among those with levels in the highest tertile of both markers $(n=$
159) relative to participants with lower levels of both markers $(n=446)$. In HIV-uninfected men, the adjusted difference in annual eGFR decline was -2.09 (95\% CI -4.38 to 0.20$) \mathrm{mL} / \mathrm{min} / 1.73 \mathrm{~m}^{2}$ per year faster among those with levels in the highest tertiles of both markers $(n=61)$ relative to participants with lower levels of both markers $(n=174)$.

Because both tenofovir exposure and HIV viremia can impact kidney tubular health, we then evaluated whether TDF use and detectable HIV RNA levels at baseline mod- 
Table 3. Association of urine biomarkers with annual eGFR change in HIV- MACS participants

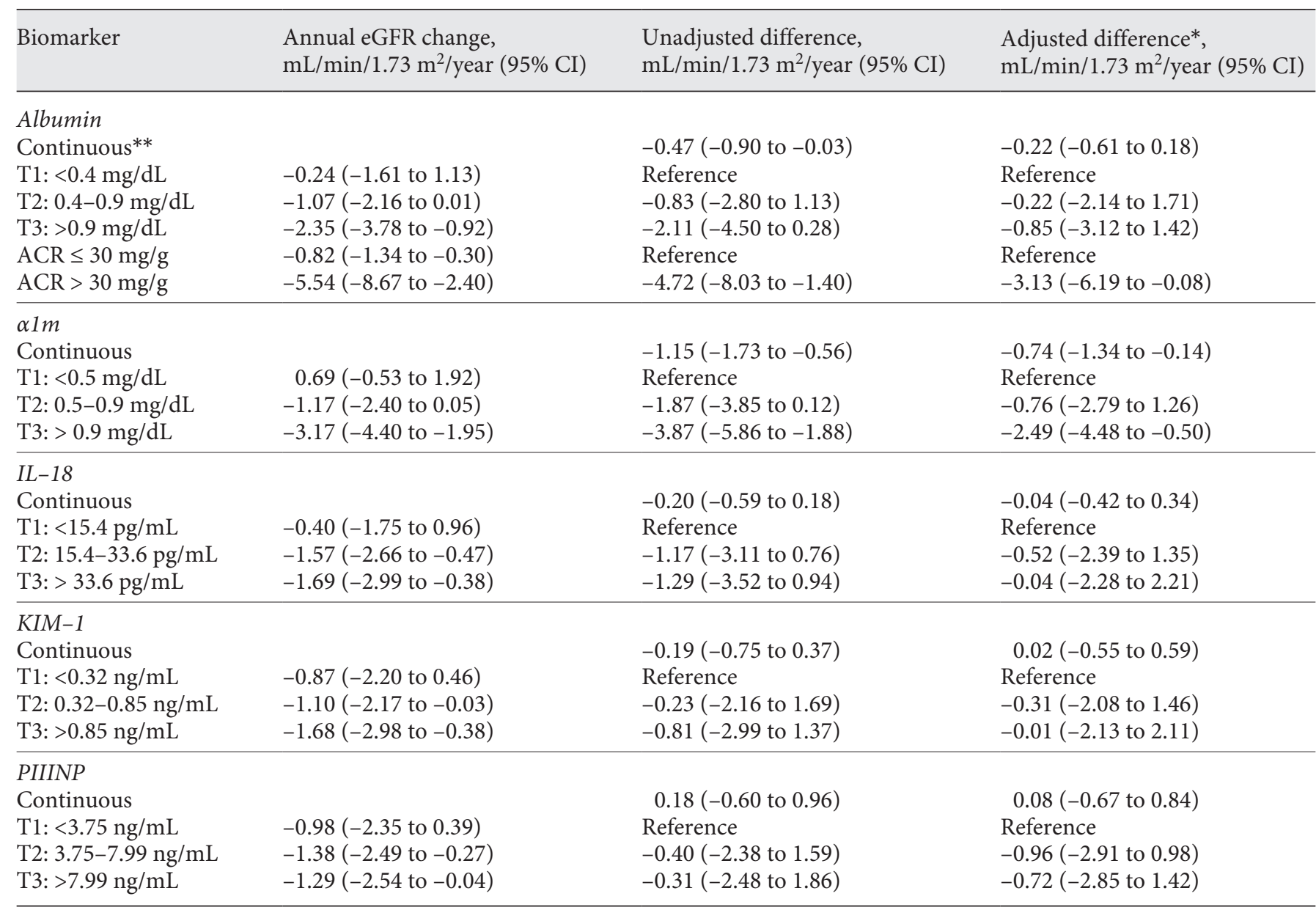

Biomarkers modeled individually, not jointly.

* Models adjust for age, race/ethnicity, urine creatinine, baseline eGFR, diabetes mellitus, systolic and diastolic blood pressure, hypertension, cardiovascular disease, low- and high-density lipoprotein cholesterol levels, triglyceride level, body mass index, waist circumference, cigarette smoking status, serum albumin, current heroin use, HCV infection.

** Continuous predictors are modeled per two-fold higher level of biomarker at baseline.

ACR, albumin-to-creatinine ratio; alm, alpha-1-microglobulin; eGFR, estimated glomerular filtration rate; IL-18, interleukin-18; KIM-1, kidney injury molecule-1; PIIINP, procollagen type III N-terminal propeptide; HCV, hepatitis C virus.

ified the associations of the urine biomarkers with changes in eGFR in HIV-infected participants. Each two-fold higher urine albumin and IL-18 at baseline were associated with faster eGFR declines among non-TDF users compared to TDF users, whereas associations of urine a1m, KIM-1, and PIIINP with eGFR decline did not differ by TDF use (online suppl. Table 3). Higher urine biomarker levels were associated with faster eGFR declines in men with detectable HIV RNA levels compared to men with undetectable HIV RNA, although differences reached statistical significance only for IL-18 $(p=0.023)$. Each two-fold higher IL-18 at baseline was associated with $-0.39 \mathrm{~mL} / \mathrm{min} / 1.73 \mathrm{~m}^{2}$ per year $(95 \% \mathrm{CI}-1.02$ to $0.24)$ faster eGFR decline among men with detectable HIV RNA levels and $+0.42 \mathrm{~mL} / \mathrm{min} / 1.73 \mathrm{~m}^{2}$ per year (95\% CI 0.05-0.79) slower eGFR decline among those with undetectable HIV RNA.

Among HIV-infected participants, 98 (12\%) developed incident CKD and 155 (18\%) had an annual eGFR decline $\geq 5 \%$ over the approximately 5 years of follow-up. In unadjusted analyses, higher baseline urine albumin and $a 1 \mathrm{~m}$ concentrations were individually associated with risks of both incident CKD and $\geq 5 \%$ annual eGFR decline (Table 4). However, in multivariable models that 
adjusted for baseline eGFR, the associations were attenuated and none remained statistically significant.

Among HIV-uninfected participants, 23 (6.8\%) developed incident CKD and $22(6.8 \%)$ had an annual eGFR decline $\geq 5 \%$ during follow-up. In unadjusted analyses, only higher levels of urine albumin were associated with risk of incident CKD (risk ratio per two-fold higher urine albumin: 1.34, 95\% CI 1.00-1.79), but this association was substantially attenuated and not statistically significant after multivariable adjustment including adjustment for baseline eGFR. None of the urine biomarkers were significantly associated with risk of $\geq 5 \%$ annual eGFR decline among HIV-uninfected participants; unadjusted point estimates were similar to those observed in the HIV-infected participants.

\section{Discussion}

As the burden of chronic, noninfectious comorbidities accumulates in an aging HIV population, early and accurate identification of kidney disease has become increasingly important. In this large ambulatory cohort, higher urine albumin and $\alpha 1 \mathrm{~m}$ concentrations at baseline were individually associated with faster kidney function decline in HIV-infected and uninfected men, independent of baseline eGFR and kidney disease risk factors. When we adjusted for all biomarkers simultaneously, associations with kidney function decline remained significant for urine albumin in HIV-infected individuals and for urine alm in HIV-uninfected individuals. Participants in the highest tertiles for both urine albumin and a $1 \mathrm{~m}$ had substantially faster kidney function decline, as compared with participants in the lowest tertiles for each marker. By contrast, urine IL-18, KIM-1, and PIIINP did not show statistically significant associations with eGFR changes in either HIV-infected or uninfected participants.

The associations of higher urine albumin and $\alpha 1 \mathrm{~m} \mathrm{lev-}$ els with kidney function decline among HIV-infected men are consistent with previous studies [18, 19,27]. By contrast, the null associations with incident CKD and rapid kidney function decline may have been due to loss of information from categorizing eGFR, reduced power, or the relatively preserved kidney function of MACS participants at baseline. In the Women's Interagency HIV Study (WIHS), a large, multicenter cohort of HIV-infected women, higher urine albumin levels were associated with faster eGFR decline, and higher urine a $1 \mathrm{~m}$ levels were associated with risk of incident $\mathrm{CKD}$, independent

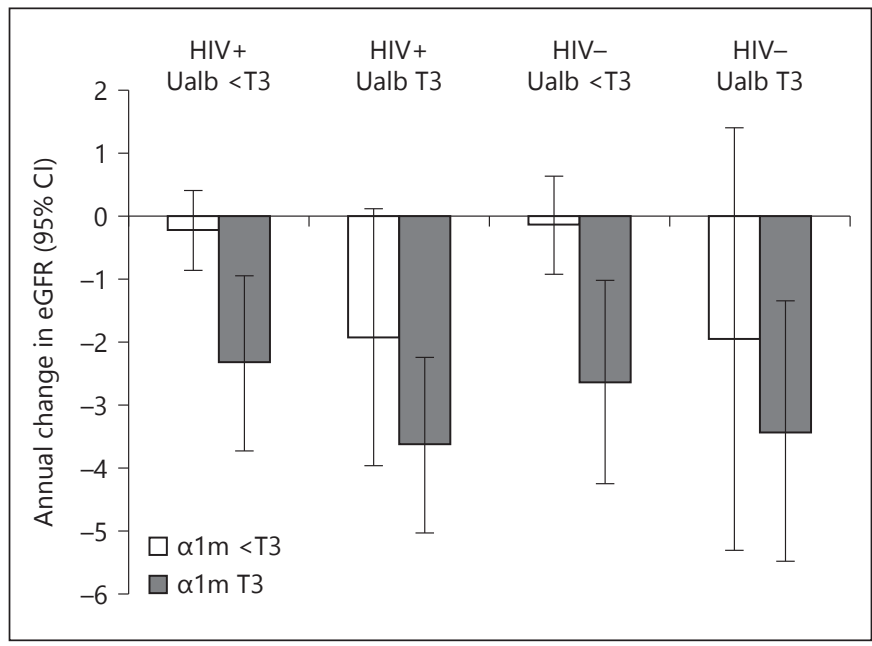

Fig. 1. Annual change in eGFR across tertiles of urine albumin and alm in HIV-infected and uninfected MACS participants. Bars represent unadjusted estimated annual change in eGFR in $\mathrm{mL} /$ $\min / 1.73 \mathrm{~m}^{2}$, with $95 \%$ CIs displayed. Estimates are compared across the highest versus lower tertiles of urine albumin and $\alpha 1 \mathrm{~m}$ and are derived from linear mixed effect models. $\alpha 1 \mathrm{~m}$, alpha-1-microglobulin; eGFR, estimated glomerular filtration rate; T3, highest tertile; <T3, first or second tertile; Ualb, urine albumin.

of albuminuria and baseline eGFR [18, 19]. Urine albumin primarily reflects glomerular injury and is an established risk factor for CKD progression [28]. Our results support current HIV guidelines that recommend monitoring for kidney damage with albuminuria [7] and suggest further investigation into potential use of urine $a 1 \mathrm{~m}$ for risk stratification in future studies. In contrast to albumin, $\alpha 1 \mathrm{~m}$ is a freely filtered, low-molecular-weight protein that is fully reabsorbed by the proximal tubule; elevated levels in urine indicate dysfunction of the proximal tubule [29]. Our data suggest that albuminuria and urine $a 1 \mathrm{~m}$ may provide complementary information about subsequent risk of declining kidney function, but further work is needed to validate these findings.

Similar to several previous studies in the general population, our study found no significant associations of urine IL-18, KIM-1, or PIIINP levels with eGFR decline [30-33]. IL-18 is a proinflammatory cytokine released by proximal tubular cells in response to injury or inflammation, while KIM-1 is upregulated and overexpressed by dedifferentiated proximal tubular cells after injury [34, 35]. Urine PIIINP reflects the presence of type III collagen deposits in the renal interstitium during fibrosis, and higher urine PIIINP concentrations correlate with increased severity of fibrosis on kidney biopsy $[36,37]$. We hypothesized that higher urine IL-18, KIM-1, and PIIINP 
Table 4. Association of urine biomarkers with rapid eGFR decline and incident CKD in HIV+ MACS participants

\begin{tabular}{lllr}
\hline & $\begin{array}{l}\text { Unadjusted } \\
\text { RR }(95 \% \text { CI })\end{array}$ & $\begin{array}{l}\text { Multivariable- } \\
\text { adjusted RR (95\% CI)* }\end{array}$ & $\begin{array}{l}\text { Multivariable-adjusted + } \\
\text { eGFR RR (95\% CI)* }\end{array}$ \\
\hline 5\% annual eGFR decline $(n=155 / 860)$ & & & $1.11(0.98-1.25)$ \\
$\quad$ Albumin & $1.16(1.03-1.30)$ & $1.12(1.00-1.27)$ & $1.09(0.97-1.23)$ \\
alm & $1.13(1.01-1.28)$ & $1.12(0.99-1.26)$ & $1.10(0.92-1.32)$ \\
IL-18 & $1.17(0.96-1.43)$ & $1.10(0.92-1.33)$ & $1.00(0.85-1.19)$ \\
KIM-1 & $1.03(0.86-1.23)$ & $1.00(0.84-1.19)$ & $1.00(0.85-1.17)$ \\
PIIINP & $1.05(0.89-1.25)$ & $1.03(0.87-1.21)$ & $1.08(0.96-1.22)$ \\
Incident CKD $(n=98 / 786)$ & $1.18(1.04-1.34)$ & $1.16(0.99-1.35)$ & $1.09(0.93-1.27)$ \\
Albumin & $1.23(1.08-1.40)$ & $1.17(1.01-1.35)$ & $1.11(0.90-1.37)$ \\
alm & $1.11(0.91-1.35)$ & $1.06(0.87-1.30)$ & $1.03(0.85-1.26)$ \\
IL-18 & $1.11(0.92-1.35)$ & $1.00(0.81-1.23)$ & $1.04(0.88-1.24)$ \\
KIM-1 & $1.17(0.96-1.43)$ & $1.10(0.90-1.35)$ & \\
PIIINP & &
\end{tabular}

Biomarkers are modeled per two-fold higher level at baseline and are modeled individually, not jointly.

* Models adjust for age, race/ethnicity, urine creatinine, diabetes mellitus, systolic and diastolic blood pressure, hypertension, cardiovascular disease, low- and high-density lipoprotein cholesterol levels, triglyceride level, body mass index, waist circumference, cigarette smoking status, serum albumin, current heroin use, HCV infection, current CD4 lymphocyte count, plasma HIV-1 RNA level, history of clinical AIDS diagnosis, TDF use, and highly active antiretroviral therapy use.

alm, alpha-1-microglobulin; eGFR, estimated glomerular filtration rate; IL-18, interleukin-18; RR, risk ratio; KIM-1, kidney injury molecule-1; PIIINP, procollagen type III N-terminal propeptide; HCV, hepatitis C virus; CKD, chronic kidney disease; MACS, Multicenter AIDS Cohort Study.

levels would be associated with kidney function decline because tubulointerstitial injury and fibrosis are strong predictors of CKD progression [9, 10, 13, 14]. Contrary to our findings, higher urine IL-18 and KIM-1 levels among HIV-infected and uninfected participants of the WIHS were independently associated with larger eGFR declines [18]. Additionally, a cohort study of middleaged, ethnically diverse men and women also showed higher levels of KIM-1 were associated with an increased risk of incident CKD [38]. Among ambulatory older adults, higher urine PIIINP levels have been associated with the risk of CKD progression [39]. The discrepancy between our findings and previous work in HIV-infected individuals could be explained by differences in the study population. By comparison to the WIHS, our study consisted of men who were older, had higher prevalence of diabetes mellitus and hypertension, lower prevalence of smoking and hepatitis C, and higher prevalence of HIV viral suppression. Additional studies will be required to determine the optimal subset of urinary biomarkers for prognostication and monitoring of kidney health among HIV-infected individuals.

Strengths of our study include the use of a multicenter, diverse, contemporary cohort representative of HIV-infected men in the United States with a follow-up period of approximately 5 years, assessment of levels of multiple biomarkers of kidney damage, and inclusion of a similar group of HIV-uninfected men. Our study also has several limitations. First, we were unable to estimate GFR using serum cystatin Clevels, which may be a more sensitive indicator of early losses in kidney function [4]. Second, urine biomarkers were measured once at baseline, so we were unable to evaluate changes in biomarker levels over time. Repeated biomarkers may produce stronger associations with kidney-related outcomes that are several years after the exposure. Third, our observational study may not have accounted for all potential confounders given the numerous risk factors for kidney disease, although these models were comprehensively constructed with numerous potential confounders. Third, our results are not generalizable to HIV-infected women or to HIV-infected individuals not on antiretroviral therapy.

In summary, we observed that higher levels of urine albumin and $\alpha 1 \mathrm{~m}$, a marker of kidney tubule dysfunction, were associated with faster declines in kidney function in a cohort of HIV-infected and uninfected men. Further studies are needed to determine the utility of monitoring levels of biomarkers of kidney tubular health, in addition to eGFR and albuminuria, among persons living with HIV. 


\section{Acknowledgments}

The authors thank the participants and staff of the MACS, which has centers at Baltimore (U01-AI35042): The Johns Hopkins University Bloomberg School of Public Health: Joseph B. Margolick (PI), Jay Bream, Todd Brown, Barbara Crain, Adrian Dobs, Richard Elion, Richard Elion, Michelle Estrella, Lisette Johnson-Hill, Sean Leng, Anne Monroe, Cynthia Munro, Michael W. Plankey, Wendy Post, Ned Sacktor, Jennifer Schrack, Chloe Thio; Chicago (U01-AI35039): Feinberg School of Medicine, Northwestern University, and Cook County Bureau of Health Services: Steven M. Wolinsky (PI), John P. Phair, Sheila Badri, Dana Gabuzda, Frank J. Palella, Jr., Sudhir Penugonda, Susheel Reddy, Matthew Stephens, Linda Teplin; Los Angeles (U01-AI35040): University of California, UCLA Schools of Public Health and Medicine: Roger Detels (PI), Otoniel Martínez-Maza (Co-P I), Aaron Aronow, Peter Anton, Robert Bolan, Elizabeth Breen, Anthony Butch, Shehnaz Hussain, Beth Jamieson, Eric N. Miller, John Oishi, Harry Vinters, Dorothy Wiley, Mallory Witt, Otto Yang, Stephen Young, Zuo Feng Zhang; Pittsburgh (U01-AI35041): University of Pittsburgh, Graduate School of Public Health: Charles R. Rinaldo (PI), Lawrence A. Kingsley (Co-PI), James T. Becker, Phalguni Gupta, Kenneth Ho, Susan Koletar, Jeremy J. Martinson, John W. Mellors, Anthony J. Silvestre, Ronald D. Stall; Data Coordinating Center (UM1-AI35043): The Johns Hopkins University Bloomberg School of Public Health: Lisa P. Jacobson (PI), Gypsyamber D'Souza (Co-PI), Alison, Abraham, Keri Althoff, Jennifer Deal, Priya Duggal, Sabina Haberlen, Alvaro Muoz, Derek Ng, Janet Schollenberger, Eric C. Seaberg, Sol Su, Pamela Surkan. Institute of Allergy and Infectious Diseases: Robin E. Huebner; National Cancer Institute: Geraldina Dominguez. The MACS is funded primarily by the National Institute of Allergy and Infectious Diseases, with additional co-funding from the National Cancer Institute, the National Institute on Drug Abuse, and the National Institute of Mental Health. Targeted supplemental funding for specific projects was also provided by the National Heart, Lung, and Blood Institute and the National Institute on Deafness and Communication Disorders (NIDCD). MACS data collection is also supported by UL1-TR001079 (JHU ICTR) from the National Center for Advancing Translational Sciences a component of the National Institutes of Health (NIH) and NIH Roadmap for Medical Research. The contents of this publication are solely the respon- sibility of the authors and do not represent the official views of the NIH, Johns Hopkins ICTR, or National Center for Advancing Translational Sciences. The funders of this study had no role in study design; collection, analysis or interpretation of data; manuscript preparation; or the decision to submit the report for publication. The MACS website is located at http://www.statepi.jhsph. $\mathrm{edu} / \mathrm{macs} / \mathrm{macs}$. html.

\section{Statement of Ethics}

The Institutional Review Boards of participating institutions approved the study protocol (IRB \#10-00827), and informed consent was obtained from all study participants. The University of California, San Francisco and San Francisco Veterans Affairs Medical Center committees on human research approved this study.

\section{Disclosure Statement}

None declared. The results presented in this paper have not been published previously in whole or part, except in abstract format.

\section{Funding Sources}

This work was supported by the NIA (R01AG034853 for M.G.S./C.R.P.) and NIDDK (K23DK109868 for V.J.).

\section{Author Contributions}

S.B.A., R.S., M.M.E., M.G.S., and V.K.J. are responsible for the research idea and study design. D.K.N. and F.J.P. are responsible for data acquisition. S.B.A., R.S., M.M.E., M.G.S., V.K.J., D.K.N., F.J.P., M.D.W., K.H., M.R.B., C.R.P., and J.H.I. are responsible for data analysis and interpretation. R.S. is responsible for statistical analysis. V.K.J. is responsible for supervision and mentorship.

\section{References}

1 Antiretroviral Therapy Cohort Collaboration. Life expectancy of individuals on combination antiretroviral therapy in high-income countries: a collaborative analysis of 14 cohort studies. Lancet. 2008 Jul;372(9635):293-9.

2 Palella FJ Jr, Baker RK, Moorman AC, Chmiel JS, Wood KC, Brooks JT, et al.; HIV Outpatient Study Investigators. Mortality in the highly active antiretroviral therapy era: changing causes of death and disease in the HIV outpatient study. J Acquir Immune Defic Syndr. 2006 Sep;43(1):27-34.

3 Eggers PW, Kimmel PL. Is there an epidemic of HIV Infection in the US ESRD pro- gram? J Am Soc Nephrol. 2004 Sep;15(9): 2477-85.

4 Odden MC, Scherzer R, Bacchetti P, Szczech LA, Sidney S, Grunfeld C, et al. Cystatin C level as a marker of kidney function in human immunodeficiency virus infection: the FRAM study. Arch Intern Med. 2007 Nov;167(20): 2213-9.

5 Estrella MM, Parekh RS, Astor BC, Bolan R, Evans RW, Palella FJ Jr, et al. Chronic kidney disease and estimates of kidney function in HIV infection: a cross-sectional study in the multicenter AIDS cohort study. J Acquir Immune Defic Syndr. 2011 Aug;57(5):380-6.
6 Choi AI, Li Y, Deeks SG, Grunfeld C, Volberding PA, Shlipak MG. Association between kidney function and albuminuria with cardiovascular events in $\mathrm{HIV}$-infected persons. Circulation. 2010 Feb;121(5):651-8.

7 Lucas GM, Ross MJ, Stock PG, Shlipak MG, Wyatt CM, Gupta SK, et al.; HIV Medicine Association of the Infectious Diseases Society of America. Clinical practice guideline for the management of chronic kidney disease in patients infected with HIV: 2014 update by the HIV Medicine Association of the Infectious Diseases Society of America. Clin Infect Dis. 2014 Nov;59(9):e96-138. 
8 Driver TH, Scherzer R, Peralta CA, Tien PC, Estrella MM, Parikh CR, et al. Comparisons of creatinine and cystatin $\mathrm{C}$ for detection of kidney disease and prediction of all-cause mortality in HIV-infected women. AIDS. 2013 Sep;27(14):2291-9.

9 Kassirer JP. Clinical evaluation of kidney function-glomerular function. $\mathrm{N}$ Engl J Med. 1971 Aug;285(7):385-9.

10 Nath KA. Tubulointerstitial changes as a major determinant in the progression of renal damage. Am J Kidney Dis. 1992 Jul;20(1):117.

11 Winston JA, Bruggeman LA, Ross MD, Jacobson J, Ross L, D’Agati VD, et al. Nephropathy and establishment of a renal reservoir of HIV type 1 during primary infection. N Engl J Med. 2001 Jun;344(26):1979-84.

12 Ross MJ, Bruggeman LA, Wilson PD, Klotman PE. Microcyst formation and HIV-1 gene expression occur in multiple nephron segments in HIV-associated nephropathy. J Am Soc Nephrol. 2001 Dec;12(12):2645-51.

13 Wyatt CM, Morgello S, Katz-Malamed R, Wei C, Klotman ME, Klotman PE, et al. The spectrum of kidney disease in patients with AIDS in the era of antiretroviral therapy. Kidney Int. $2009 \mathrm{Feb} ; 75(4): 428-34$.

14 Rule AD, Amer H, Cornell LD, Taler SJ, Cosio FG, Kremers WK, et al. The association between age and nephrosclerosis on renal biopsy among healthy adults. Ann Intern Med. 2010 May;152(9):561-7.

15 Jotwani V, Scherzer R, Abraham A, Estrella MM, Bennett M, Devarajan P, et al. Does HIV infection promote early kidney injury in women? Antivir Ther. 2014;19(1):79-87.

16 Jotwani V, Scherzer R, Estrella MM, Jacobson LP, Witt MD, Palella FJ Jr, et al. HIV Infection, Tenofovir, and Urine a1-Microglobulin: A Cross-sectional Analysis in the Multicenter AIDS Cohort Study. Am J Kidney Dis. 2016 Oct;68(4):571-81.

17 Jotwani V, Scherzer R, Estrella MM, Jacobson LP, Witt MD, Palella F, et al. Association of HIV infection with biomarkers of kidney injury and fibrosis in the Multicenter AIDS Cohort Study. Antivir Ther. 2017;22(5):421-9.

18 Shlipak MG, Scherzer R, Abraham A, Tien PC, Grunfeld C, Peralta CA, et al. Urinary markers of kidney injury and kidney function decline in HIV-infected women. J Acquir Immune Defic Syndr. 2012 Dec;61(5): $565-73$.

19 Jotwani V, Scherzer R, Abraham A, Estrella MM, Bennett M, Cohen MH, et al. Associa- tion of urine a1-microglobulin with kidney function decline and mortality in HIV-infected women. Clin J Am Soc Nephrol. 2015 Jan; 10(1):63-73.

20 Kaslow RA, Ostrow DG, Detels R, Phair JP, Polk BF, Rinaldo CR Jr. The Multicenter AIDS Cohort Study: rationale, organization, and selected characteristics of the participants. Am J Epidemiol. 1987 Aug;126(2): 310-8.

21 Levey AS, Stevens LA, Schmid CH, Zhang YL, Castro AF 3rd, Feldman HI, et al.; CKD-EPI (Chronic Kidney Disease Epidemiology Collaboration). A new equation to estimate glomerular filtration rate. Ann Intern Med. 2009 May;150(9):604-12.

22 Little RJ. Pattern-Mixture Models for Multivariate Incomplete Data. J Am Stat Assoc. 1993;88(421):125-34.

23 Molenberghs G, Kenward M. Missing Data in Clinical Studies. John Wiley \& Sons; 2007. pp. 529.

24 van Buuren S. Flexible Imputation of Missing Data [Internet]. Chapman and Hall/CRC; 2012 [cited 2018 Aug 23]. Available from: https:// www.taylorfrancis.com/ books/9781439868256.

25 Fuller WA. Introduction to Statistical Time Series. New York: John Wiley \& Sons; 1976.

26 Lumley T, Kronmal R, Ma S. Relative Risk Regression in Medical Research: Models, Contrasts, Estimators, and Algorithms [Internet]. UW Biostat Work Pap Ser [2006 Jul 19]. Available from: https://biostats.bepress.com/ uwbiostat/paper293.

27 Ando M, Yanagisawa N, Ajisawa A, Tsuchiya K, Nitta K. Kidney tubular damage in the absence of glomerular defects in HIV-infected patients on highly active antiretroviral therapy. Nephrol Dial Transplant. 2011 Oct; 26(10):3224-9.

28 Gansevoort RT, Matsushita K, van der Velde M, Astor BC, Woodward M, Levey AS, et al.; Chronic Kidney Disease Prognosis Consortium. Lower estimated GFR and higher albuminuria are associated with adverse kidney outcomes. A collaborative meta-analysis of general and high-risk population cohorts. Kidney Int. 2011 Jul;80(1):93-104

29 Weber MH, Verwiebe R. Alpha 1-microglobulin (protein HC): features of a promising indicator of proximal tubular dysfunction. Eur J Clin Chem Clin Biochem. 1992 Oct;30(10): 683-91.

30 Bhavsar NA, Köttgen A, Coresh J, Astor BC. Neutrophil gelatinase-associated lipocalin
(NGAL) and kidney injury molecule 1 (KIM1 ) as predictors of incident CKD stage 3: the Atherosclerosis Risk in Communities (ARIC) Study. Am J Kidney Dis. 2012 Aug;60(2):23340.

31 O'Seaghdha CM, Hwang SJ, Larson MG, Meigs JB, Vasan RS, Fox CS. Analysis of a urinary biomarker panel for incident kidney disease and clinical outcomes. J Am Soc Nephrol. 2013 Nov;24(11):1880-8.

32 Fufaa GD, Weil EJ, Nelson RG, Hanson RL, Bonventre JV, Sabbisetti V, et al.; Chronic Kidney Disease Biomarkers Consortium Investigators. Association of urinary KIM-1, LFABP, NAG and NGAL with incident endstage renal disease and mortality in American Indians with type 2 diabetes mellitus. Diabetologia. 2015 Jan;58(1):188-98.

33 Nadkarni GN, Rao V, Ismail-Beigi F, Fonseca VA, Shah SV, Simonson MS, et al. Association of Urinary Biomarkers of Inflammation, Injury, and Fibrosis with Renal Function Decline: the ACCORD Trial. Clin J Am Soc Nephrol. 2016 Aug;11(8):1343-52.

34 Parikh CR, Jani A, Melnikov VY, Faubel S, Edelstein CL. Urinary interleukin-18 is a marker of human acute tubular necrosis. Am J Kidney Dis. 2004 Mar;43(3):405-14.

35 Han WK, Bailly V, Abichandani R, Thadhani $\mathrm{R}$, Bonventre JV. Kidney Injury Molecule-1 (KIM-1): a novel biomarker for human renal proximal tubule injury. Kidney Int. 2002 Jul; 62(1):237-44.

36 Ghoul BE, Squalli T, Servais A, Elie C, MeasYedid V, Trivint C, et al. Urinary procollagen III aminoterminal propeptide (PIIINP): a fibrotest for the nephrologist. Clin J Am Soc Nephrol. 2010 Feb;5(2):205-10.

37 Soylemezoglu O, Wild G, Dalley AJ, MacNeil S, Milford-Ward A, Brown CB, et al. Urinary and serum type III collagen: markers of renal fibrosis. Nephrol Dial Transplant. 1997 Sep; 12(9):1883-9.

38 Peralta CA, Katz R, Bonventre JV, Sabbisetti $\mathrm{V}$, Siscovick D, Sarnak M, et al. Associations of urinary levels of kidney injury molecule 1 (KIM-1) and neutrophil gelatinase-associated lipocalin (NGAL) with kidney function decline in the Multi-Ethnic Study of Atherosclerosis (MESA). Am J Kidney Dis. 2012 Dec; 60(6):904-11.

39 Ix JH, Biggs ML, Mukamal K, Djousse L, Siscovick D, Tracy R, et al. Urine Collagen Fragments and CKD Progression-The Cardiovascular Health Study. J Am Soc Nephrol. 2015 Oct;26(10):2494-503. 